\title{
The association of homeobox gene expression with stem cell formation and morphogenesis in cultured Medicago truncatula
}

\author{
S.-K. Chen $\cdot$ S. Kurdyukov $\cdot$ A. Kereszt $\cdot$ X.-D. Wang $\cdot$ \\ P. M. Gresshoff $\cdot$ R. J. Rose
}

Received: 20 February 2009 / Accepted: 9 July 2009 / Published online: 29 July 2009

(C) The Author(s) 2009. This article is published with open access at Springerlink.com

\begin{abstract}
Somatic embryogenesis (SE) is induced in vitro in Medicago truncatula 2HA by auxin and cytokinin but rarely in wild type Jemalong. The putative WUSCHEL (MtWUS), CLAVATA3 (MtCLV3) and the WUSCHELrelated homeobox gene WOX5 (MtWOX5) were investigated in $M$. truncatula $(M t)$ and identified by the similarity to Arabidopsis WUS, CLV3 and WOX5 in amino acid sequence, phylogeny and in planta and in vitro expression patterns. MtWUS was induced throughout embryogenic cultures by cytokinin after $24-48 \mathrm{~h}$ and maximum expression occurred after 1 week, which coincides with the induction of totipotent stem cells. During this period there was no $M t C L V 3$ expression to suppress MtWUS. MtWUS expression, as illustrated by promoter-GUS studies, subsequently localised to the embryo, and there was then the onset of $M t C L V 3$ expression. This suggests that the expression of the putative MtCLV3 coincides with the WUS-CLAVATA feedback loop becoming operational. RNAi studies showed
\end{abstract}

Electronic supplementary material The online version of this article (doi:10.1007/s00425-009-0988-1) contains supplementary material, which is available to authorized users.

S.-K. Chen $\cdot$ S. Kurdyukov $\cdot$ X.-D. Wang $\cdot$ R. J. Rose $(\varangle)$

School of Environmental and Life Sciences,

Australian Research Council Centre of Excellence for Integrative

Legume Research, The University of Newcastle,

Callaghan, NSW 2308, Australia

e-mail: Ray.Rose@newcastle.edu.au

A. Kereszt · P. M. Gresshoff

Australian Research Council Centre of Excellence for Integrative Legume Research, The University of Queensland,

Brisbane, QLD 4072, Australia

Present Address:

A. Kereszt

Baygen Institute, 6726 Szeged, Hungary that MtWUS expression is essential for callus and somatic embryo production. Based on the presence of MtWUS promoter binding sites, MtWUS may be required for the induction of MtSERF1, postulated to have a key role in the signalling required for SE induced in 2HA. MtWOX5 expressed in auxin-induced root primordia and root meristems and appears to be involved in pluripotent stem cell induction. The evidence is discussed that the homeobox genes MtWUS and MtWOX5 are "hijacked" for stem cell induction, which is key to somatic embryo and de novo root induction. In relation to SE, a role for WUS in the signalling involved in induction is discussed.

Keywords Medicago $\cdot$ Root development $\cdot$ Somatic embryogenesis - Stem cell formation - WUSCHEL . WUSCHEL-related homeobox genes

$\begin{array}{ll}\text { Abbreviations } \\ \text { Aux } & \text { Auxin } \\ \text { CLE } & \text { CLV3/endosperm surrounding region } \\ \text { CLV3 } & \text { CLAVATA3 } \\ \text { Cyt } & \text { Cytokinin } \\ \text { EST } & \text { Expressed sequence tag } \\ \text { GUS } & \beta \text {-Glucuronidase } \\ \text { RNAi } & \text { RNA interference } \\ \text { SAM } & \text { Shoot apical meristem } \\ \text { SE } & \text { Somatic embryogenesis } \\ \text { WOX } & \text { WUSCHEL-related homeobox } \\ \text { WUS } & \text { WUSCHEL }\end{array}$

\section{Introduction}

The homeobox gene WUSCHEL (WUS) encodes a homeodomain transcription factor that has been shown to be a 
regulator of a pool of pluripotent stem cells in the apical meristem (Mayer et al. 1998; Bäurle and Laux 2005; Reddy and Meyerowitz 2005; Shani et al. 2006). Zuo et al. (2002) identified gain-of-function mutants, which caused somatic embryo formation in Arabidopsis in a range of tissues and organs. The responsible gene was found to be identical to WUS. Overexpression of WUS could induce somatic embryogenesis (SE). Zuo et al. (2002) concluded that WUS had a key role in the vegetative-to-embryogenic transition and in addition to having its well-known role in meristem development, could act as an embryo organiser. Gallois et al. (2004) have also investigated ectopic WUS expression and found that shoots could be induced in the roots and somatic embryos in the presence of auxin. This suggested that pluripotent or totipotent cells could be induced by WUS depending on the hormonal environment.

In the shoot meristem WUS expression is regulated by the small protein CLAVATA3 (CLV3) (Brand et al. 2000; Fiers et al. 2007). As the population of stem cells increases there is an increase in the synthesis and secretion of CLV3, which subsequently causes a decrease in the population of stem cells (Beveridge et al. 2007). CLV3 is proposed to bind to the CLV1/CLV2 receptor complex, initiating a signalling cascade, which leads to down-regulation of WUS expression in the cells of the organiser region of the apical meristem (Brand et al. 2000; Fiers et al. 2007; Ogawa et al. 2008). However there is no direct biochemical evidence for CLV3 interaction with a CLV1/CLV2 receptor complex as opposed to a CLV1/CLV1 complex. Müller et al. (2008) have evidence that the novel receptor kinase CORYNE and CLV2 may act together, and in parallel with CLV1 homodimers to perceive the CLV3 signal.

If overexpression of WUS can induce somatic embryos, then it would be expected that a similar result could be achieved by preventing the CLV signalling. Mordhorst et al. (1998), using the primordia timing (pt), clv1 and $c l v 3$ mutants and $p t c l v$ double mutants, found a correlation between increased shoot apical meristem size and an increased frequency of seedlings producing embryogenic seed lines.

There is a family of transcription factors related to WUS known as the WUSCHEL-related homeobox (WOX) gene family that includes WOX5 (Haecker et al. 2004). In Arabidopsis WOX5 is expressed in quiescent cells of the root apical meristem (Haecker et al. 2004). Stem cells surround the quiescent centre (Scheres 2005). Investigations by Sarkar et al. (2007) have shown that WOX5 acts to maintain the stem cells of the root apex and can be considered analogous to WUS that acts to maintain stem cells of the shoot apex. WOX5 expression occurs during in vitro root formation in cultured Medicago truncatula (Imin et al. 2007).

Medicago truncatula is a model legume (Rose 2008) that has been used to investigate the mechanisms of in vitro somatic embryo (Rose and Nolan 2006) and root formation
(Rose et al. 2006; Imin et al. 2007). When leaf explants were cultured on basal medium with auxin and cytokinin, somatic embryos were induced and when cultured on basal medium plus auxin then roots were produced (Nolan et al. 2003).

The ability of plant cells to be directed into different developmental pathways in vitro provides systems that can be utilised to improve the understanding of plant stem cell biology. In this study we have further investigated the transcription factors WUSCHEL and WOX5 of M. truncatula that are important regulators of stem cell maintenance in vivo in relation to the induction of SE and root formation in vitro. The data obtained are consistent with a role for homeobox genes in the production of stem cells to produce either embryos or roots depending on the hormonal environment.

\section{Materials and methods}

\section{Plant material}

The M. truncatula cultivars Jemalong 2HA (2HA) and wild type Jemalong were grown in standard potting mix under glasshouse conditions. 2HA is a highly embryogenic mutant of Jemalong produced in our laboratory (Rose et al. 1999). The nature of the mutation is not known. We have suggested that the difference between Jemalong and 2HA is likely to be epigenetic (Rose 2008). The wild type Jemalong used was an accession (SA1619) from the Australian National Medicago collection, South Australian Research and Development Institute (SARDI), Adelaide. Cultured M. truncatula leaf explants were obtained from glasshousegrown plants.

\section{Cultured leaf explants}

The standard leaf culture procedure and media were as described by Nolan et al. (2003). Explants were cultured on P4 10:4 for 3 weeks before transfer to P4 10:4:1 (10 $\mu \mathrm{M}$ NAA, $4 \mu \mathrm{M}$ BAP and $1 \mu \mathrm{M}$ ABA added at 3 weeks). In some experiments $1 \mu \mathrm{M}$ ABA was added at the beginning of culture in the P4 10:4:1 medium as we have found recently that this increases embryo number. Other treatments used $10 \mu \mathrm{M}$ NAA alone or $4 \mu \mathrm{M}$ BAP alone.

Sequence analysis and construction of phylogenetic trees

Multiple alignment analyses were performed with $\mathrm{Clu}$ stalW using the Clustal 2.0.8 software in Clustal default colours. Phylogenetic trees were constructed using the bootstrap neighbour-joining method (1,000 rounds) (Saitou and Nei 1987) included in the Clustal 2.0.8 software. Phylogenetic trees were drawn using TreeView (Win32) 1.6.0 software (Page 1996). 
Real-time PCR

Total RNA was isolated from intact leaves as a calibrator and from calli and other tissues, using the Qiagen RNeasy Plant Mini Prep Kit (Qiagen Pty Ltd, Doncaster, VIC, Australia) as per the manufacturer's instructions. cDNA synthesis was performed using the Superscript II ${ }^{\mathrm{TM}}$ First-Strand Synthesis System for RT-PCR (Invitrogen, Carlsbad, CA, USA) starting with $1 \mu \mathrm{g}$ of total RNA with oligo (dT) 15 primers. Real-time PCR was performed using $\mathrm{SYBR}^{\circledR}$ GreenER $^{\text {TM }}$ qPCR SuperMix Universal Kit (Invitrogen) and analysed in the DNA Engine Opticon ${ }^{\circledR} 2$ Continuous Fluorescence Detection System (Bio-Rad, Gladesville, NSW, Australia). Primers designed to quantify the expression levels for $M t W U S$ were 5'-CTTACAACATTTCATCTG CTGGGCT-3' (forward) and 5'-CGACATGATGACCA ATCCATCCTAT-3' (reverse), for MtWOX5 were 5'-CAAG CACTGATCAAATTCAGAAAAT-3' (forward) and 5'-GA AAAAGCTCAAGAGTCTCAATCAC-3' (reverse), and for MtCLV3 were 5'-ATGGCTTCTAAGTTCATCTTTTC TT-3' (forward) and 5' -TCAAGGGTTTTCAGGCTTAA TAGGG-3' (reverse), which were normalised to those of glyceraldehyde-3-phosphate dehydrogenase (GAPDH), primers 5'-TGGTCATCAAACCCTCAACA-3' (forward) and 5'-CCTCGTTCTTTCCGCTATCA-3' (reverse), in each sample every run. The tubes were then cycled at $94^{\circ} \mathrm{C}$ for $30 \mathrm{~s}$, annealed at $60^{\circ} \mathrm{C}$ for $60 \mathrm{~s}$, and extended at $72^{\circ} \mathrm{C}$ for $60 \mathrm{~s}$. A melting curve was generated at the end of every run to ensure product uniformity. PCR reactions were performed in triplicate in at least two biological repeats. Transcript abundance was estimated using a modification of the comparative threshold cycle $(\mathrm{Ct})$ method and was calculated as $E^{-\Delta \Delta \mathrm{Ct}}$, where $\Delta \Delta \mathrm{Ct}=\left(\mathrm{Ct}_{\text {target }}-\mathrm{Ct}_{\mathrm{GAPDH}}\right)_{\text {Time } x}-$ $\left(\mathrm{Ct}_{\text {target }}-\mathrm{Ct}_{\mathrm{GAPDH}}\right)_{\text {Calibrator }}$ and $E$ is the estimated amplification efficiency, which was calculated employing the linear regression method on the $\log$ (fluorescence) per cycle number data for each amplicon using the LinRegPCR software (Ramakers et al. 2003).

In-situ hybridisation

To generate the RNA probes, a 893-bp fragment of MtWUS was first amplified by PCR with the primers $5^{\prime}$-ATGGAA CAGCCTCAACAACAACAA-3' (forward) and 5'-GGTG ACCTACAGCCGTAAGAGTTGA-3' (reverse). Then, the promoter sequences of T7 and SP6 RNA polymerase were introduced to this fragment by a two-step PCR. The first primers used were 5'-GAGGCCGCGTATGGAACAGCC TCAACAACA-3' (forward) and 5'-ACCCGGGGCTGGT GACCTACAGCCGTAAGA-3' (reverse). The second set of primers used were 5'-TTATGTAATACGACTCACT ATAGGGAGGCCGCGT-3' (forward) and 5'-CCAATTT AGGTGACACTATAGAAGTACCCGGGGCT-3' (reverse).
For $M t W O X 5$, a 659-bp fragment of full length cDNA sequence was first amplified by PCR with primers $5^{\prime}-\mathrm{G}$ TAAAAACATCTAGAATTGAAATATGG-3' (forward) and 5'-TCCTAAACATTTTTCATATTATGCT-3' (reverse). Sites for T7 and SP6 RNA polymerase were introduced through two-step PCR as for MtWUS. The first primers used were 5'-GAGGCCGCGTGTAAAAACATCTAG AATTGA-3' (forward) and 5'-ACCCGGGGCTTCCTA AACATTTTTCATATT-3' (reverse). The second set of primers was the same as for MtWUS. This PCR product was subsequently used as a template for in vitro transcription employing T7 and SP6 RNA polymerase to synthesise digoxigenin-labelled sense and anti-sense single-stranded RNA probes respectively using a DIG RNA Labelling Kit (Roche, Basel, Switzerland). Four- to five-week-old calli and other tissues were fixed in $4 \%$ formaldehyde in $0.025 \mathrm{M}$ phosphate buffer at $\mathrm{pH} 7.2$, dehydrated through an ethanol and ethanol: histolene (Fronine, Lomb Scientific, Taren Point, NSW, Australia) series, embedded in paraffin, sectioned, and hybridised with the digoxigenin-labelled sense and anti-sense probes as described previously (Mantiri et al. 2008a, b). The hybridisation was detected using a Fluorescent Antibody Enhancer Set for DIG detection (Roche) and was visualised as a red/purple colour after the NBT/BCIP colour reaction (Roche). Sense-strand probes were used as controls.

Construction of promoter-GUS fusions and inducible RNAi plasmids

For MtWUS promoter::GUS construction, a 3,182-bp fragment of promoter region was amplified by PCR with the primers 5'-CTAACTTCCGTTATCCGAGAATCTT-3' (forward) and 5'-TGTTCCATGTTTTTGTTGGACTGAA-3' (reverse). For $M t W U S$ RNAi construction, a 204-bp fragment was amplified by PCR with the primers 5'-CTTAC AACATTTCATCTGCTGGGCT-3' (forward) and 5'-CG ACATGATGACCAATCCATCCTAT-3' (reverse). For MtWOX5 promoter::GUS construction, a 1,024-bp fragment of promoter region was amplified by PCR with the primers $5^{\prime}$-TTCCCAACATAATTTGTAACCTCAT-3' (forward) and 5'-CATGCTCTCTTCCATATTTCAATTC-3' (reverse). For the empty vector control, 88 bp of DNA was taken from the multiple cloning site of the vector pASKIBA44, (5'-CCGGGGATCCCTCGAGGTCGACCTGCAG GGGGACCATGGTCTCAGGCCTGAGAGGATCGCATC ACCATCACCATCACTAATAAGCTT-3') (IBA, Göttingen, Germany). The gene-specific PCR products were cloned into the vector $\mathrm{pCR} 8 / \mathrm{GW} / \mathrm{TOPO}$ (Invitrogen). After extraction of the plasmids, the Gateway LR recombination reaction (Invitrogen) was carried out according to the manufacturer's protocol to insert the gene-specific fragment into the binary T-DNA destination vector pMDC164 
for promoter-GUS fusion constructs (Curtis and Grossniklaus 2003) or pOpOff2(hyg) (Wielopolska et al. 2005) for inducible RNAi constructs. The resulting constructs were introduced into Agrobacterium tumefaciens strain AGL1 by electroporation.

\section{Transformation of M. truncatula}

Transformation of $M$. truncatula 2HA leaf explants was carried out as described by Wang et al. (1996). The leaf explant preparation procedure was as described by Nolan et al. (2003). The 2HA sterilised explants were dipped into the Agrobacterium suspension and co-cultured on agar medium and incubated in the dark at $26^{\circ} \mathrm{C}$ for $2-3$ days. The explants were washed with sterilised water and $500 \mu \mathrm{g} \mathrm{ml}^{-1}$ timentin before placing on P4 10:4 solid medium plus $500 \mu \mathrm{g} \mathrm{ml}^{-1}$ augmentin and $15 \mu \mathrm{g} \mathrm{ml}^{-1}$ hygromycin and incubated in the dark at $27^{\circ} \mathrm{C}$. Hygromycin was used for transformed callus selection. The explants were subcultured every 4 weeks until somatic embryo development. For the RNAi studies transformed callus was used and RNAi constructs were induced by $2.5 \mu \mathrm{M}$ dexamethasone.

For the MtWOX5 promoter::GUS studies transformation was carried out as above, but with auxin alone as the plant hormone to produce transformed roots.

\section{Results}

The Medicago truncatula WUS and WOX5 orthologs

Using the Arabidopsis WUS and the WOX genes described by Haecker et al. (2004), together with WOX family genes from other species; M. truncatula WUS and 12 potential WOX genes were found in $M$. truncatula by BLAST searches on the NCBI and TIGR databases. Alignment of the homeodomain sequences was then carried out as seen in Fig. 1. The putative MtWUS homeodomain showed an $84 \%$ identity with AtWUS (and high identity with other species) and the putative MtWOX5 homeodomain showed an $89 \%$ identity with AtWOX5.

A phylogenetic analysis was then carried out with the sequences shown in Fig. 1 showing that MtWUS is in the WUS clade and MtWOX5 is in a clade that includes AtWOX5 and is most closely related to AtWOX5 (Fig. 2). After phylogenetic analysis of the homeodomains we also performed full length protein alignments against close homologs from Arabidopsis (not shown). This enabled us to conclude that we had identified putative MtWUS, MtWOX1, MtWOX3, MtWOX4, MtWOX5 and MtWOX9 genes. No MtWUS EST had been previously identified and we amplified the cDNA corresponding to the coding region.
The expression of the genes we designated MtWUS and MtWOX 5 was determined in different tissues that based on Arabidopsis studies would show different expression patterns. In the case of $M t W U S$ there was an expression pattern (Fig. 3a) consistent with the expression of AtWUS in the organiser centre of the apical meristem (Mayer et al. 1998; Bäurle and Laux 2005) and also in floral meristems (Müller et al. 2006). WUS was also expressed in the developing embryo of Arabidopsis (Mayer et al. 1998) and expression would be expected in the somatic embryo. In relation to zygotic embryos supplementary data (Supplementary Figure 1) have been provided for pods where the MtWUS expression correlates with embryogenesis. There was no WUS expression in the leaf or the auxin-induced cultured roots.

Further more detailed cellular studies were carried out using in-situ hybridisation with shoot meristems, heartstage zygotic embryos and ovules (Fig. 4a-d). In the shoot meristem and heart stage embryos WUS mRNA was localised in the centre of the shoot meristem in the third or fourth outermost cell layers similar to Arabidopsis (Mayer et al. 1998). There was hybridisation in the young ovules reflecting WUS expression as shown by the GUS studies in Arabidopsis by Bäurle and Laux (2005).

In the case of MtWOX5 there was an expression pattern consistent with what is known of AtWOX5 expression (Fig. 3b). AtWOX5 is expressed in the quiescent centre of the root meristem (Sarkar et al. 2007) and in the developing embryo (Haecker et al. 2004). MtWOX5 was expressed in cultured roots and in the somatic embryo (Fig. 3b) and in the embryogenesis stage of $M$. truncatula pods (Supplementary Figure 1). There was little if any expression of MtWOX5 in the shoot apex, developing flower or leaf (Fig. 3b). Previous work on MtWOX5 by Imin et al. (2007) in $M$. truncatula showed that the apical part of the plant root has 57 times higher MtWOX5 expression compared to the elongation zone. In-situ hybridisation of seedling root tips (Fig. 4e) showed expression of MtWOX5 in the region of the quiescent centre. The signal was however consistently weaker than that of the MtWUS signals (Fig. 4a-d).

The sequence and expression data are consistent with the MtWUS and MtWOX5 being indeed functional orthologs of AtWUS and AtWOX5. The promoter::GUS studies supported the qRT-PCR studies.

Expression dynamics of MtWUS and MtWOX 5

in relation to the induction of somatic embryogenesis and roots in culture

Given that ectopic expression of AtWUS can induce somatic embryos (Zuo et al. 2002), it was important in understanding the mechanism of induction of SE in M. truncatula to know the time course pattern of MtWUS 


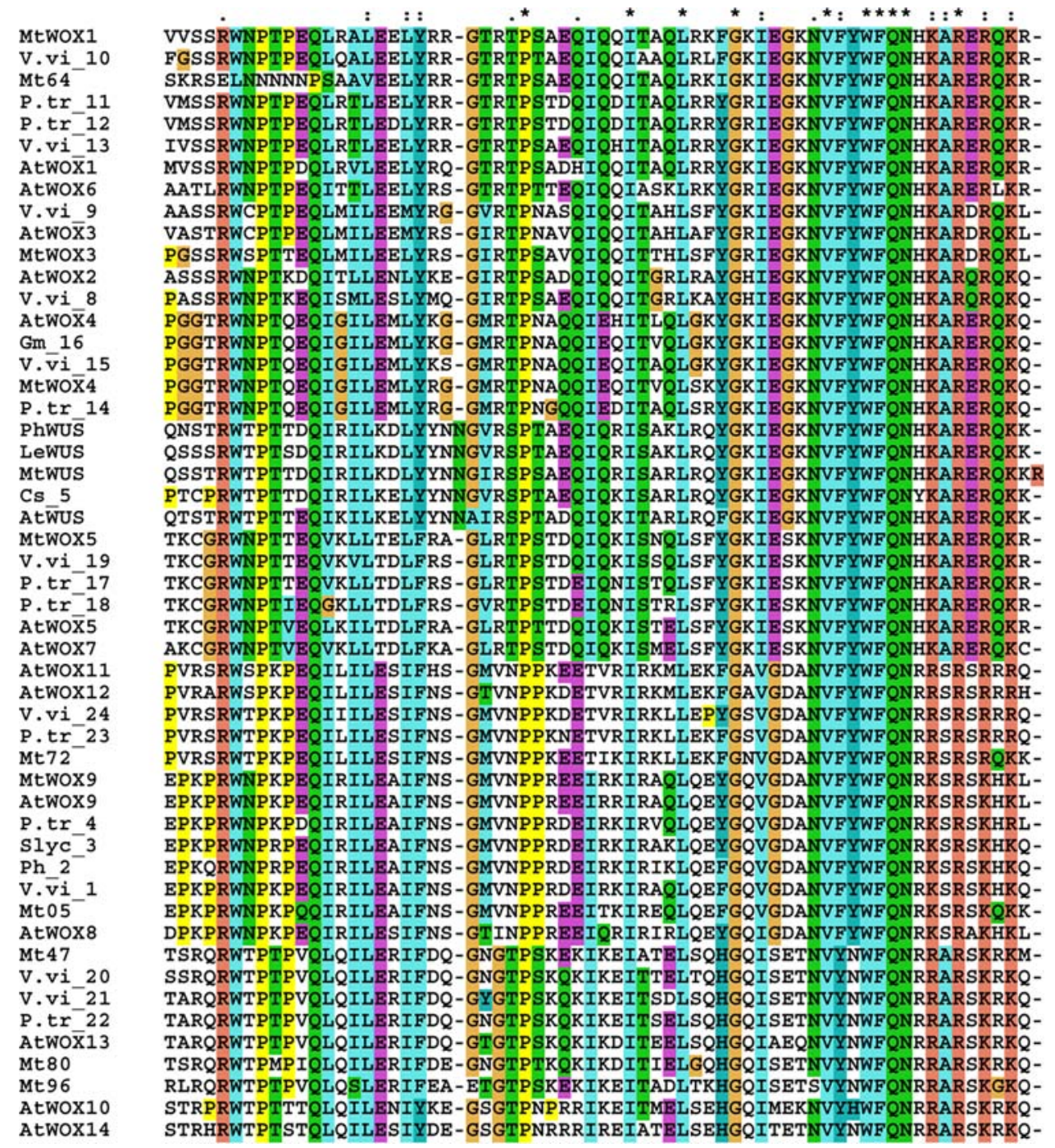

Fig. 1 Alignment of the WOX homeodomain protein sequences. Fifty-one peptide sequences from dicotyledonous species were used. At Arabidopsis thaliana, Mt Medicago truncatula MtWOX1(AC137078), MtWOX3(AC169182), MtWOX4(AC148486), MtWOX5(CU326389), MtWUS(CT009654/FJ477681), MtWOX9(AC199760), Mt64(AC14 1864), Mt80(TC104580), Mt47(BG581947), Mt96(AC232696), Mt72 (AC157472), Mt05(AC198005), Petunia (Ph) (2-EF187281, 6-PhWUS), Populus trichocarpa (P. tr)(4-AM234761, 23-AM234764, 22-AM
234762, 11-AM234756, 12-AM234757, 14-AM234759, 17-AM 234766 , 18-AM234765), Vitis Vinifera (V. vi) (13-AM439847, 10-AM 463144, 9-AM429035, 8-AM488389, 15-AM447494, 19-CAAP0 2003786, 1-AM488026, 21-AM463736, 20-AM486367, 24-AM43 5207), 3-Solanum lycopersicum (S. lyc) (FJ190667), 7-tomato(Le) LeWUS), 16-Glycine max (Gm) (DQ336954), 5-Citrus sinensis (Cs) (EU032533). Numbers at the beginning of the gene accession refer to the corresponding genes for the phylogenetic tree in Fig. 2 expression and its response to the plant hormones in the medium. In the standard auxin plus cytokinin medium MtWUS expression was induced early in the culture process (consistently within 2 days) and peaked after 7 days (Fig. 5a). The first somatic embryos were not visible to the eye until between 28 and 35 days when expression started to increase again. The increased $M t W U S$ expression was cytokinin dependent. Auxin alone did not induce MtWUS expression. MtWUS expression unlike MtWOX5 expression (see below) was not associated with in vitro root formation.

Although it was known that MtWOX5 is expressed in auxin-induced root formation in vitro (Imin et al. 2007), it remained important to understand its time course of expression to ascertain its relationship to root induction from procambial cells that we had previously described (Rose et al. 2006). MtWOX 5 expression was induced by 2 days and was clearly auxin dependent (Fig. 5b). Maximum MtWOX5 expression occurred much later when roots were visible to the unaided eye.

\section{MtWUS expression and the MtCLV3 relationship}

If MtWUS expression was associated with a process similar to that acting in the apical meristem, then one could expect 
Fig. 2 Phylogenetic tree of WOX genes. Dendrogram based on the sequence of the homeodomains. Bootstrap (1,000 rounds) was applied and the tree drawn using Dendroscope (Huson et al. 2007) with "majority" settings for consensus. Numbers and names are the same as on Fig. 1
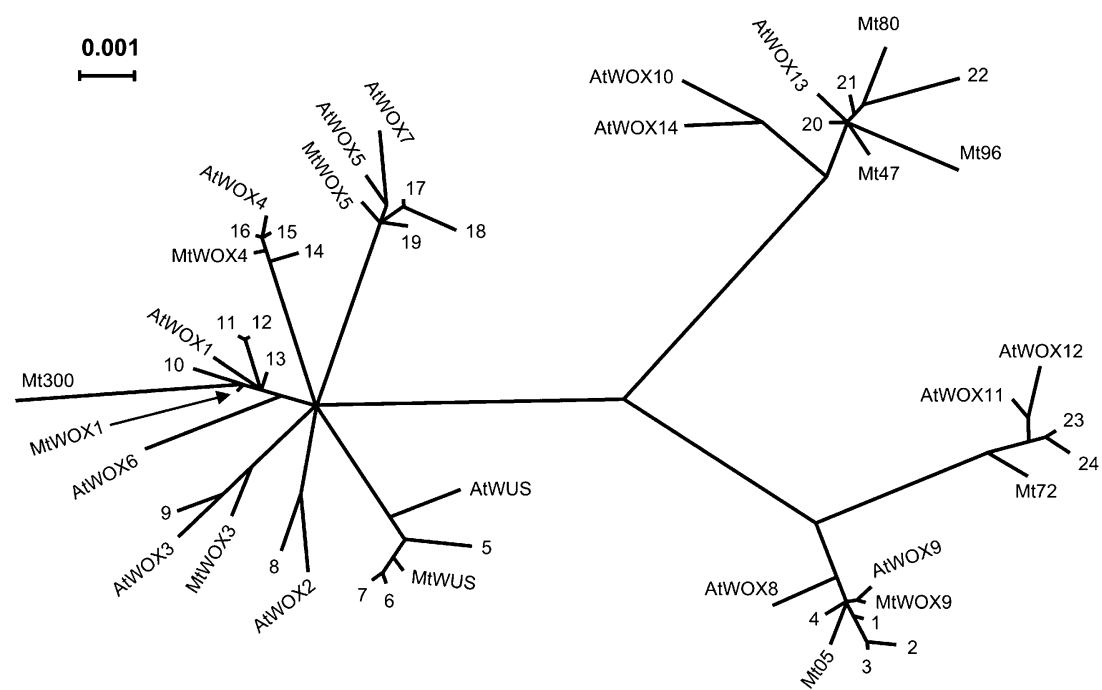

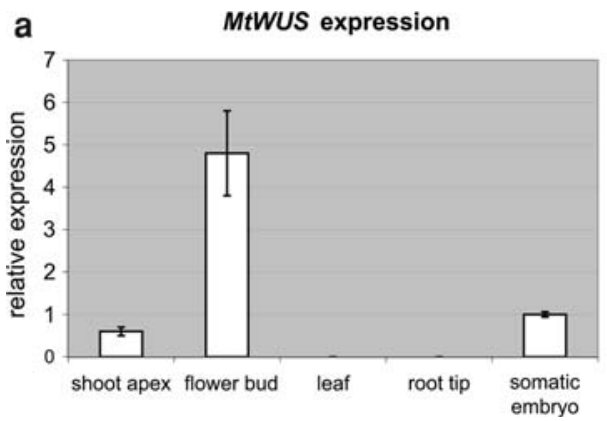

Fig. 3 Expression profiling by qRT-PCR of $M t W U S$ (a) and MtWOX5 (b) in different $M$. truncatula tissues by qRT-PCR. The expression was investigated in the shoot apices, developing flowers (buds) and mature b

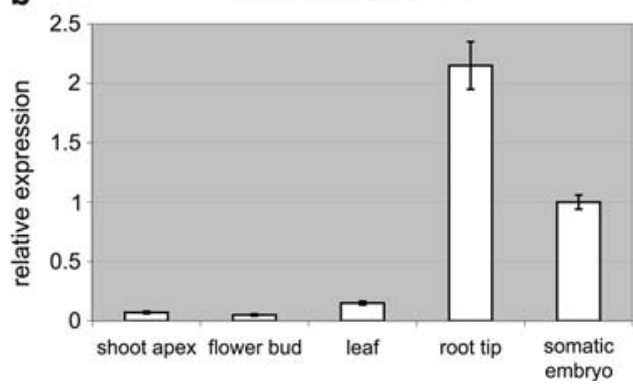

leaves of normally-grown plants, tips of cultured roots and somatic embryos (latter one in 2HA). Expression was related to the expression level of somatic embryos. Values are $\pm \operatorname{SE}(n=3)$
Fig. $4 M t W U S$ RNA in-situ hybridisation in heart stage zygotic embryos (a, b), apical meristem (c) and ovules (d) at early stage of development of wild-type Jemalong. MtWOX5 RNA in-situ hybridisation in the root meristem of a seedling root (e). $V C$ vascular cylinder (red arrows), $R C$ root cap and black arrow indicates the quiescent centre. Bar $100 \mu \mathrm{m}$ a

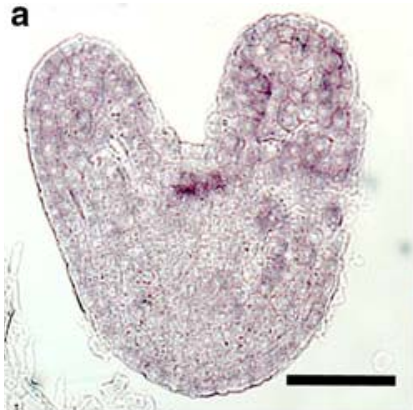

b
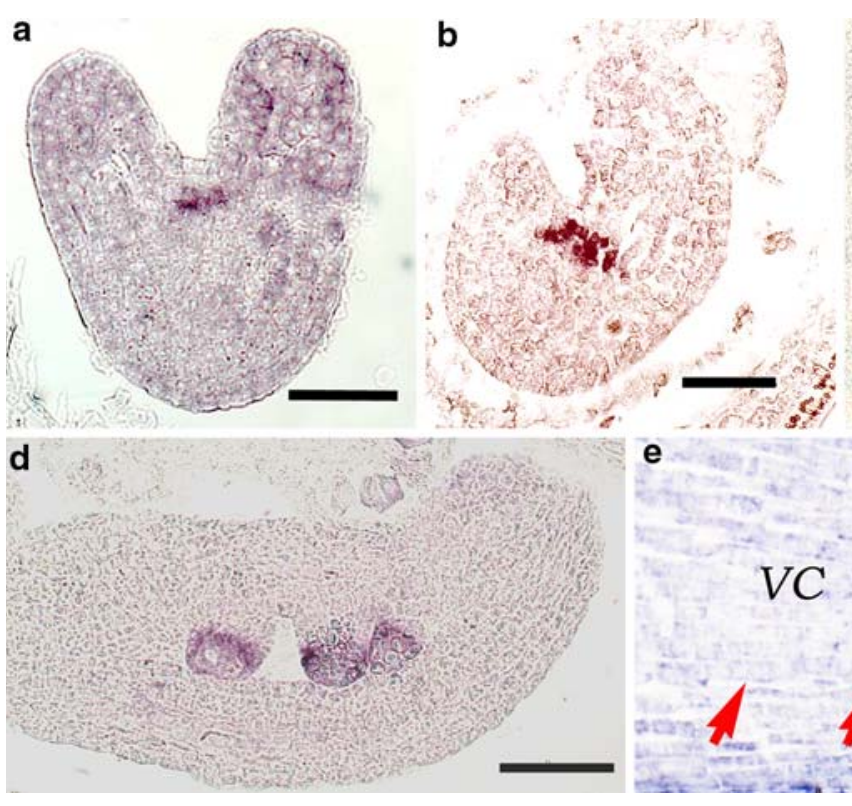

c

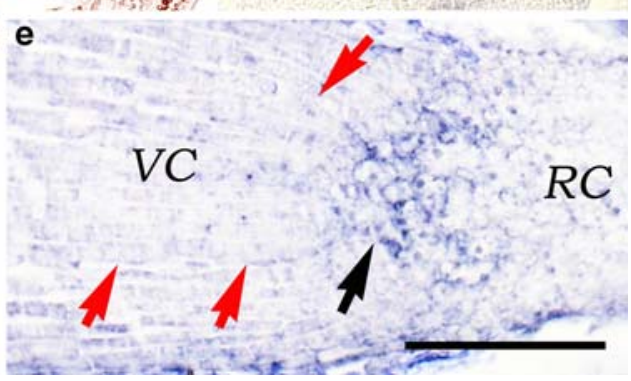

a relationship with CLV3 functionally similar to that in the apical meristem. Information obtained from Oelkers et al. (2008) and closer analysis of the Medicago MtCLE68 genomic region was used to obtain AC151522. Figure 6 shows the phylogram for the CLAVATA3/ENDOSPERM SURROUNDING REGION (CLE) peptides based on the 


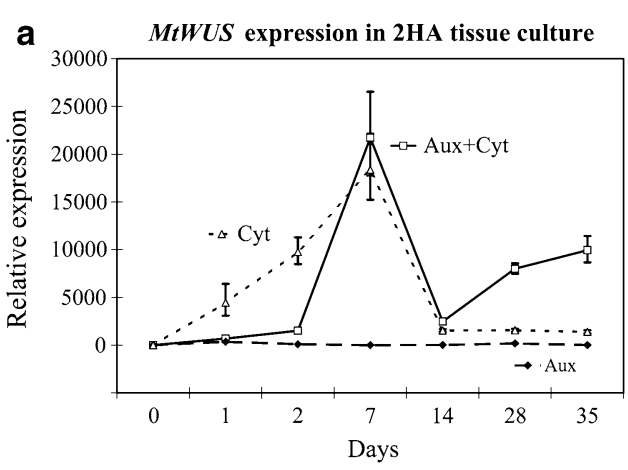

Fig. 5 Expression profiling by qRT-PCR of MtWUS (a) and MtWOX5 (b) in tissue culture with different hormones. The expression was investigated in auxin plus cytokinin (Aux $+\mathrm{Cyt}$, square-line), auxin

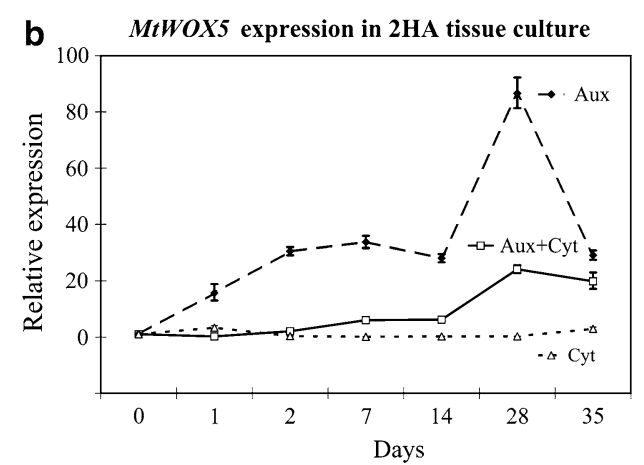

alone (Aux, diamond-dash line), and cytokinin (Cyt, triangle-dot line) treatments in 35 days in 2HA. Expression calibrated to the expression level of 0 day of $2 \mathrm{HA}$. Values are $\pm \operatorname{SE}(n=3)$
Fig. 6 Phylogram for CLEs based on the CLE domain sequence. Fifty-five genes were analysed and are detailed in Oelkers et al. (2008) [except AC151522]. Black arrow indicates the location of MtCLV3

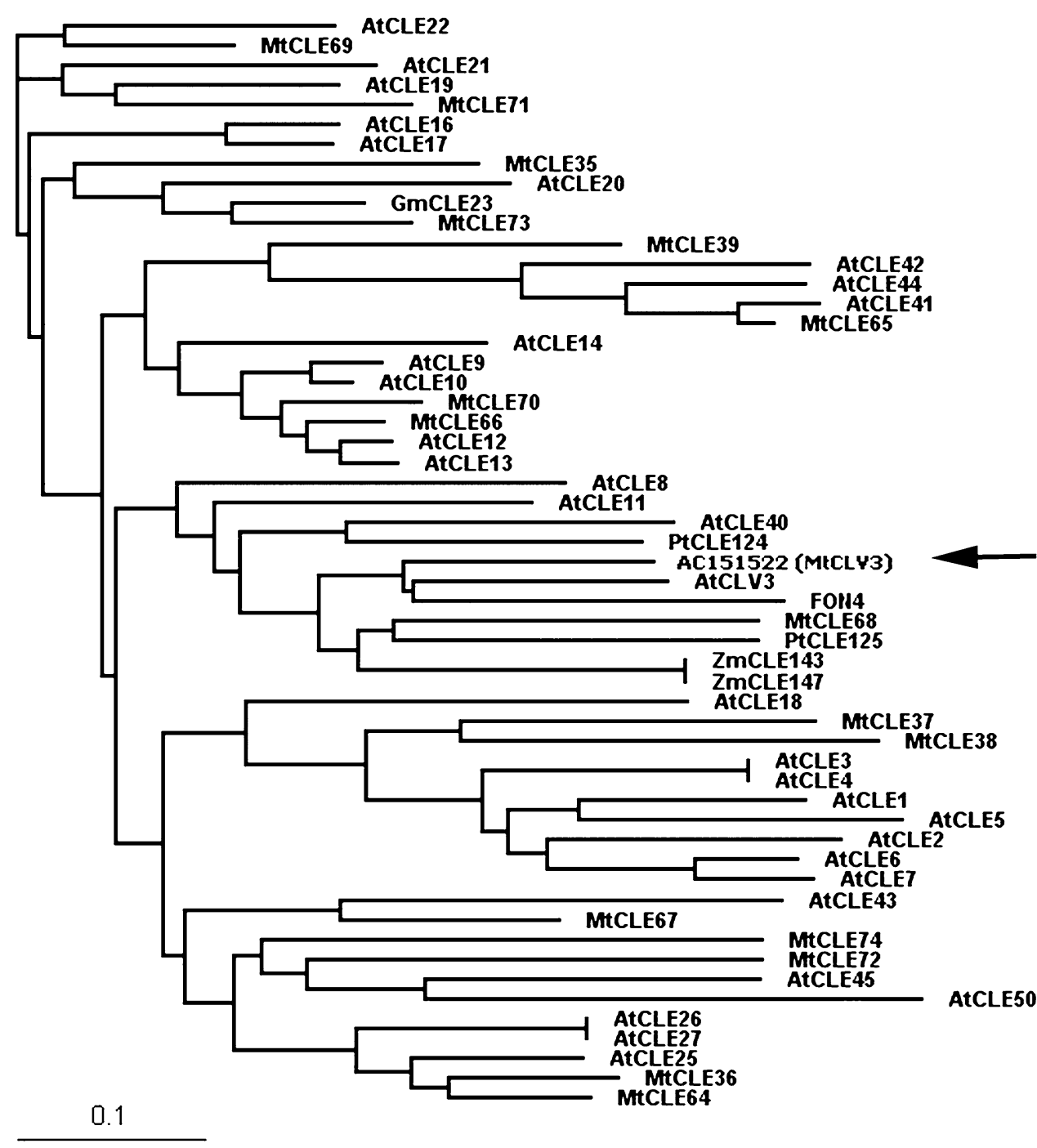

not produce embryos, did not show detectable MtCLV3 expression.

In order to investigate the role of MtWUS expression in callus formation and somatic embryo induction callus transformed with dexamethasone-inducible RNAi for entiated (Fig. 8). Importantly, wild-type Jemalong that does 


\section{MtCLV3 expression}

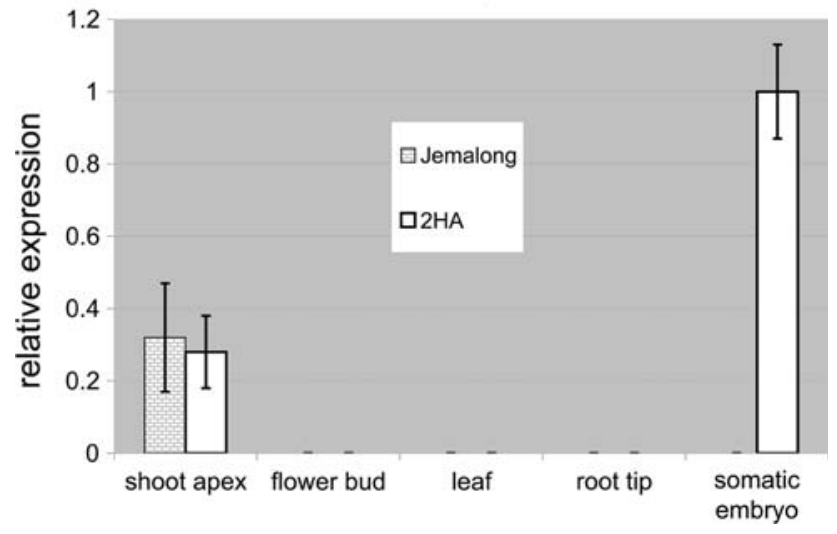

Fig.7 Expression profiling by qRT-PCR of $M t C L V 3$ in different tissues of 2HA and Jemalong. Jemalong (grey bricks) and 2HA (white). Expression normalised to the expression level in somatic embryos. Values are $\pm \operatorname{SE}(n=3)$

MtWUS was used. It was found that callus proliferation (Fig. 9) and somatic embryo induction (Fig. 10) was strongly inhibited. This suggested a role for MtWUS in both callus formation and somatic embryo induction. Further investigations on MtWUS were carried out using promoterGUS fusions and RNA in-situ hybridisation.

Using promoter-GUS fusions, MtWUS expression was consistent with the qRT-PCR data (Fig. 7). GUS expression was visualised very early in the explant and expression continued throughout the explant except at the cut edges (Fig. 11a). When strong callus growth occurred at the edges of the explant, there was strong GUS expression (Fig. 11b), but as callus developed and the explant became fully callused the expression was restricted to clusters of expression (Fig. 11c). GUS expression was present in the somatic embryos when they developed (white arrow in Fig. 11d). In regenerated transgenic $M$. truncatula plants there was GUS expression in ovules of the developing flower and later in zygotic embryogenesis. Similar results were found in

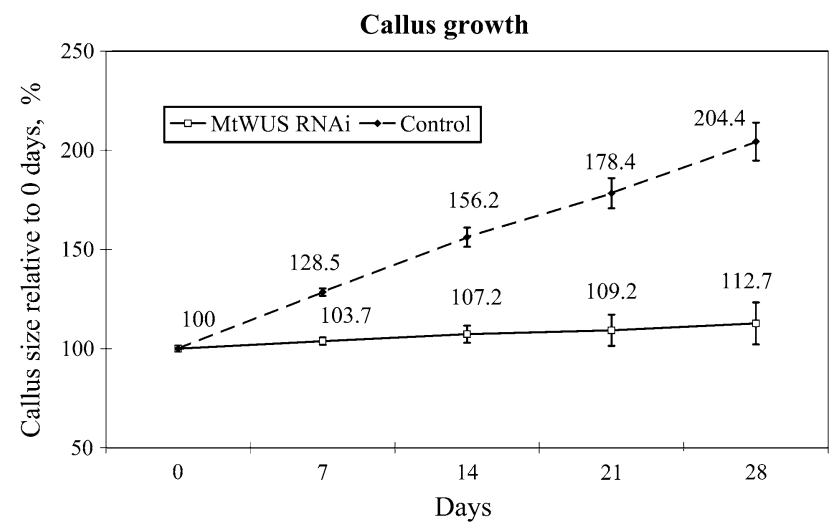

Fig. 9 The effect of dexamethasone-induced RNAi expression for MtWUS in tissue cultures (as well as an empty vector control) developed in auxin plus cytokinin medium. The callus sizes were investigated by callus imaging and are normalised with 0 day as 100 . Values are $\pm \operatorname{SE}(n=3)$

Arabidopsis (Bäurle and Laux 2005). The GUS staining at the early stage of the explant culture shown in Fig. 11a was surprising. However, cleared whole mounts stained with fuschin shows that cell division occurs throughout the explant, particularly associated with the small veins. Later in culture there is intense callus formation at the explant edges (Rose and Nolan 2006) and this is a focus of GUS expression (Fig. 11b). WUS expression in the early somatic embryos (Fig. 11d) was further investigated by in-situ hybridisation (Fig. 12). There is staining throughout the globular stage embryo and much less in the surrounding callus (Fig. 12a, b). In the embryo shown in Fig. 12c and d where the suspensor is visible, expression is greater in the top part of the embryo.

\section{MtWOX 5 expression and root meristem induction}

In-situ hybridisation studies to monitor the WOX5 expression during the formation of root primordia and
Fig. 8 Expression of $M t W U S$ and $M t C L V 3$ in tissue culture of Jemalong and 2HA lines with auxin plus cytokinin in the medium. The expressions of $M t W U S$ in 2HA (square-line) and $M t C L V 3$ in $2 \mathrm{HA}$ (diamond-dash line) and Jemalong (MtCLV3Jem, triangle-dot line) were investigated over 77 days. MtCLV3 expression only occurs in the highly embryogenic $2 \mathrm{HA}$ line as wild-type Jemalong does not produce somatic embryos. Expression normalised to the expression level of 0 day of $2 \mathrm{HA}$. Values are $\pm \mathrm{SE}(n=3)$

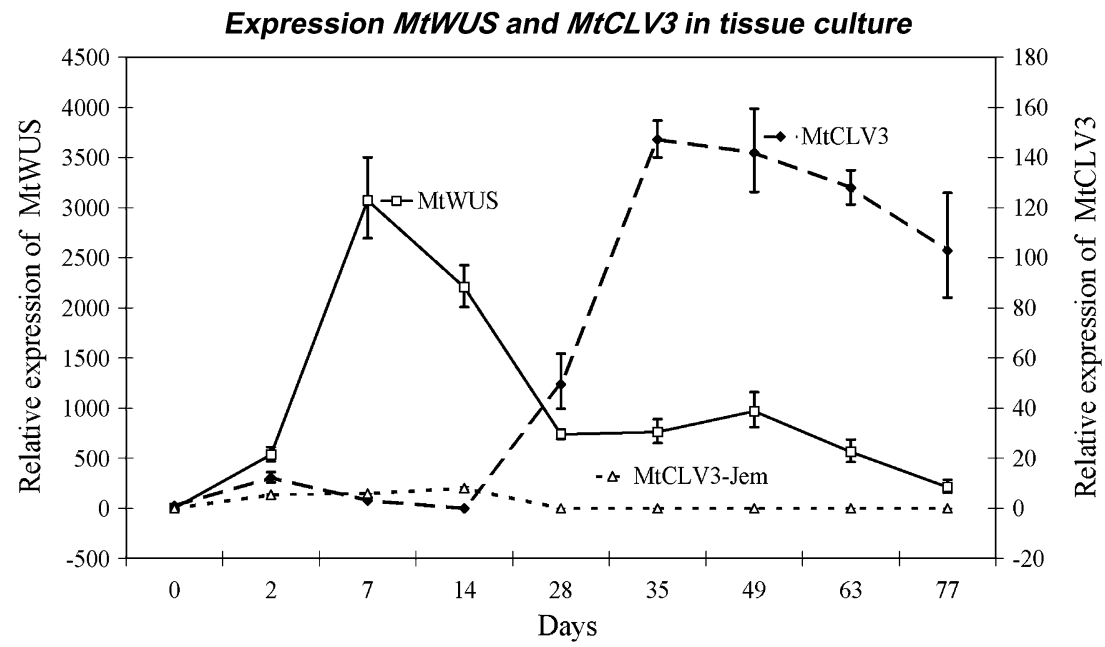


Fig. 10 Transgenic calli transformed with dexamethasoneinduced RNAi for MtWUS (a) and empty vector control (b) developed in auxin plus cytokinin culture. Somatic embryos can be seen in the control, but not in MtWUS RNAi transformed callus
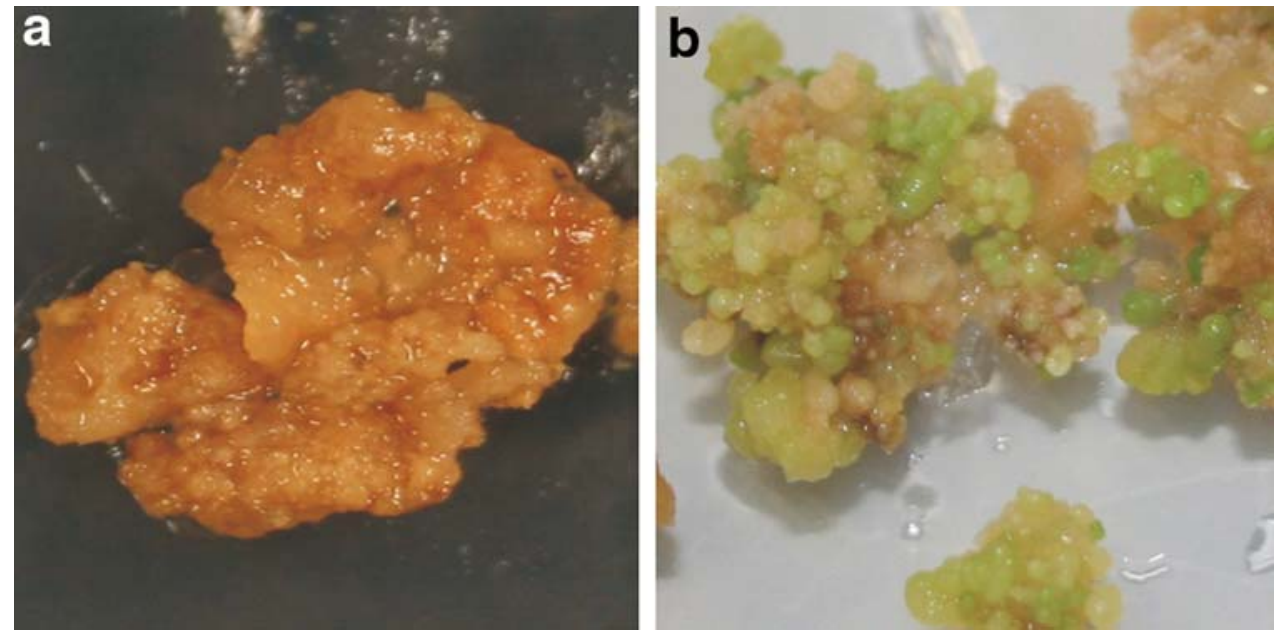
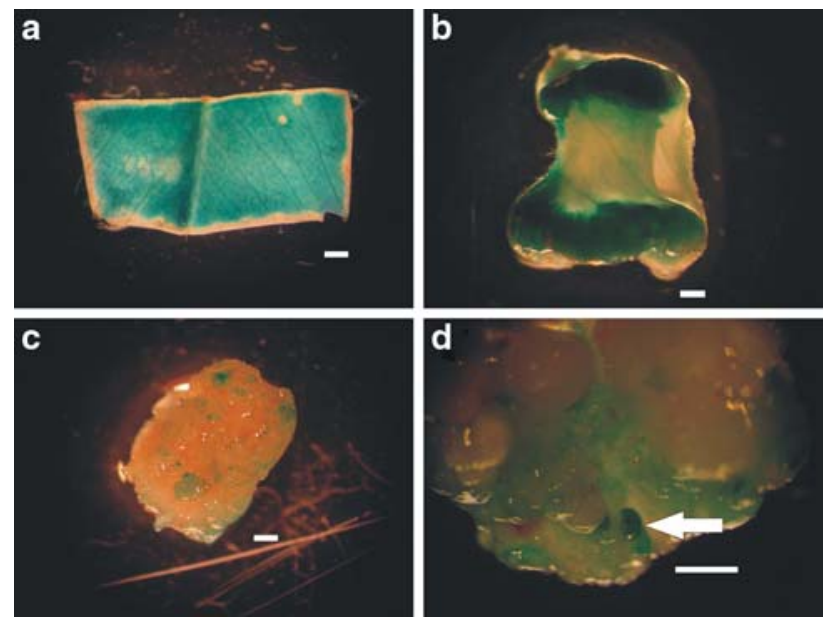

Fig. 11 MtWUS::GUS expression at the early stages of somatic embryo induction in tissue culture. Blue-green colouring indicates the GUS signal. The signals were investigated in 3 days (a), and 14 days (b) cultured explants, 28 days callus (c), and older callus (d) with somatic embryos (white arrow). Bar $500 \mu \mathrm{m}$

root meristems in the auxin-induced root formation is shown in Fig. 13. The developmental morphology has previously been documented (Rose et al. 2006). The arrow labelled 1 in Fig. 13a shows centres of expression that are what we have called vein-derived cells that emanate from the procambial cells (Rose et al. 2006). The position of the root primordium (Fig. 13a, arrow labelled 2), the root meristem (Fig. 13e, arrow labelled 3) and the vascular tissue (Fig. 13e, arrow labelled 4) are indicated.

Promoter::GUS studies (Fig. 14a) followed by sectioning of the material showed MtWOX5 expression in the stem cell areas adjacent to the quiescent centre. MtWOX5 expression can be seen in the pericycle and procambium area (Fig. 14b).

\section{Discussion}

WUSCHEL and somatic embryogenesis induction

The putative M. truncatula WUS gene ortholog was obtained using the genome sequence and isolating the cDNA. Altogether eleven WOX genes from M. truncatula were identified and five of them ascribed to particular orthologs in Arabidopsis based on protein homology; MtWOX1, MtWOX3, MtWOX4, MtWOX5 and MtWOX9.

To obtain supporting evidence that the putative MtWUS was an ortholog of AtWUS, the expression pattern of MtWUS was initially examined in the intact plant prior to studying the expression in callus formation and SE. In the intact plant, MtWUS was expressed in the shoot apex (meristem and leaf primordium), in buds, and zygotic embryos but it was not expressed in leaves or roots. The expression pattern was similar to AtWUS in the shoot meristem and the flower primordium (Bäurle and Laux 2005; Müller et al. 2006). With in-situ hybridisations gene expression was present in the same positions in the shoot apex and heart-stage embryo as in Arabidopsis (Mayer et al. 1998). It seems clear that MtWUS is the AtWUS functional ortholog.

The WUS studies presented here support the predictions from the WUS overexpression studies by Zuo et al. (2002), namely that WUS expression is an essential for SE. In the M. truncatula SE system MtWUS expression is induced in the presence of auxin and cytokinin and also cytokinin alone, but not by auxin alone. This is consistent with what is known for the WUS and cytokinin relationships in the regulation of WUS in the Arabidopsis meristem (Leibfried et al. 2005). Further, Gordon et al. (2007) have shown cytokinin-induced AtWUS expression in shoot induction in in vitro cultures. The cytokinin-induced WUS expression in M. truncatula is in itself not enough to produce SEs as wild 
Fig. $12 M t W U S$ RNA in-situ hybridisation in embryogenic callus of 2HA. The signals were investigated in whole globular stage somatic embryos. Antisense probe indicating the MtWUS signals $(\mathbf{a}, \mathbf{c})$. Sense probe controls $(\mathbf{b}, \mathbf{d})$. $\mathbf{a}$ and $\mathbf{b}$ are $40 \mu \mathrm{m}$ vibratome sections while $\mathbf{c}$ and $\mathbf{d}$ are $8 \mu \mathrm{m}$ paraffinembedded sections. The somatic embryo (c, d) has a suspensor like structure. Bar $80 \mu \mathrm{m}$
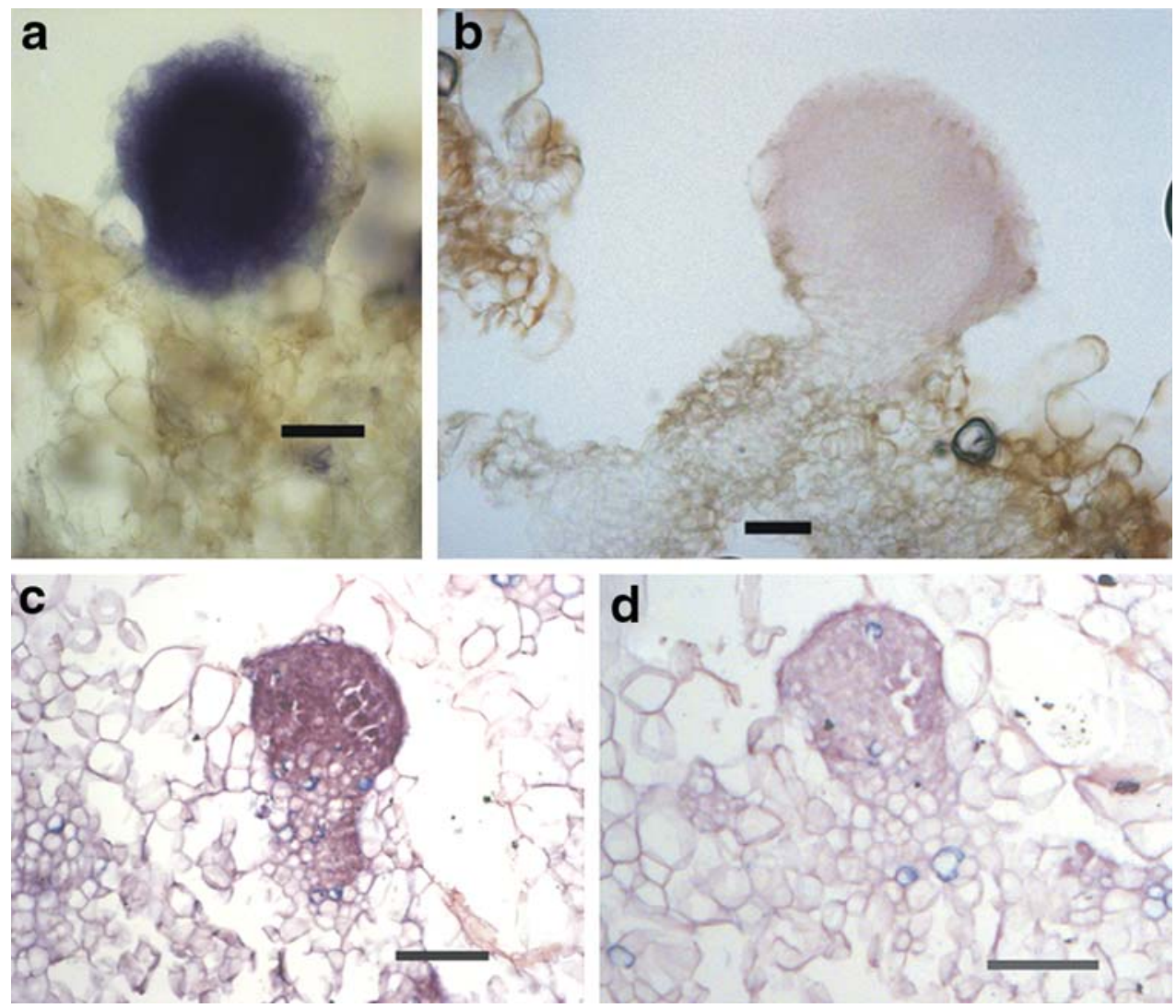

In the globular stage somatic embryo, MtWUS expression occurred throughout the whole embryo, which is not found in Arabidopsis zygotic embryos (Mayer et al. 1998). However, there are two points to note here: the hormonal environment is quite different in the somatic embryo developing in embryogenic callus, and the M. truncatula embryo is not likely to be identical to Arabidopsis in its developmental strategy. As the somatic embryo develops, MtWUS tends to localise towards the shoot pole.

The gene we have designated MtCLV3 is similar to AtCLV3 in peptide structure, genomic environment, and expression pattern. MtCLV3 also expresses in the shoot apex but not in flowers or leaves. It does not express in callus but is expressed in the shoot regions of later stage somatic embryos. MtWUS is initially expressed at high levels early in culture, unrestricted by $C L V 3$ feedback, and as $C L V 3$ is expressed it reduces WUS expression till eventually the well-known CLV3-WUS feedback loop characteristic of Arabidopsis shoot meristems is set up, i.e CLV3 down-regulates high WUS expression. Wild type Jemalong, which does not produce SEs, does not show CLV3 expression in culture.

WUS was induced in 24-48 $\mathrm{h}$ but the question remains as to how this expression relates to the overall process of SE induction. Previous work had shown that MtSERKI was expressed $48 \mathrm{~h}$ after the beginning of culture, just after MtWUS expression, and it was associated with 
Fig. $13 M t W O X 5$ RNA in-situ hybridisation during auxin-induced de novo root formation. The anti-sense probe $(\mathbf{a}-\mathbf{c})$. Sense probe for controls $(\mathbf{b}, \mathbf{d}, \mathbf{f})$. The arrow labelled "l" shows centres of expression in what we have called "vein-derived" cells that emanate from the procambial cells (Rose et al. 2006). The arrow labelled " 2 " is pointing to the root primordium. The arrow " 3 " indicates the signal in the root meristem and the arrow "4" in the vascular tissue. Bar $80 \mu \mathrm{m}$
Fig. 14 MtWOX5::GUS expression in roots induced on auxin medium. The GUS signals in the root tip (a) and root maturation zone (b) in $8 \mu \mathrm{m}$ paraffinembedded sections. Two strong areas of GUS signal are indicated by red (distal) and black (proximal) arrows in $\mathbf{a}$. In $\mathbf{b}$, the GUS signal is indicated by a red arrow. Bar $80 \mu \mathrm{m}$
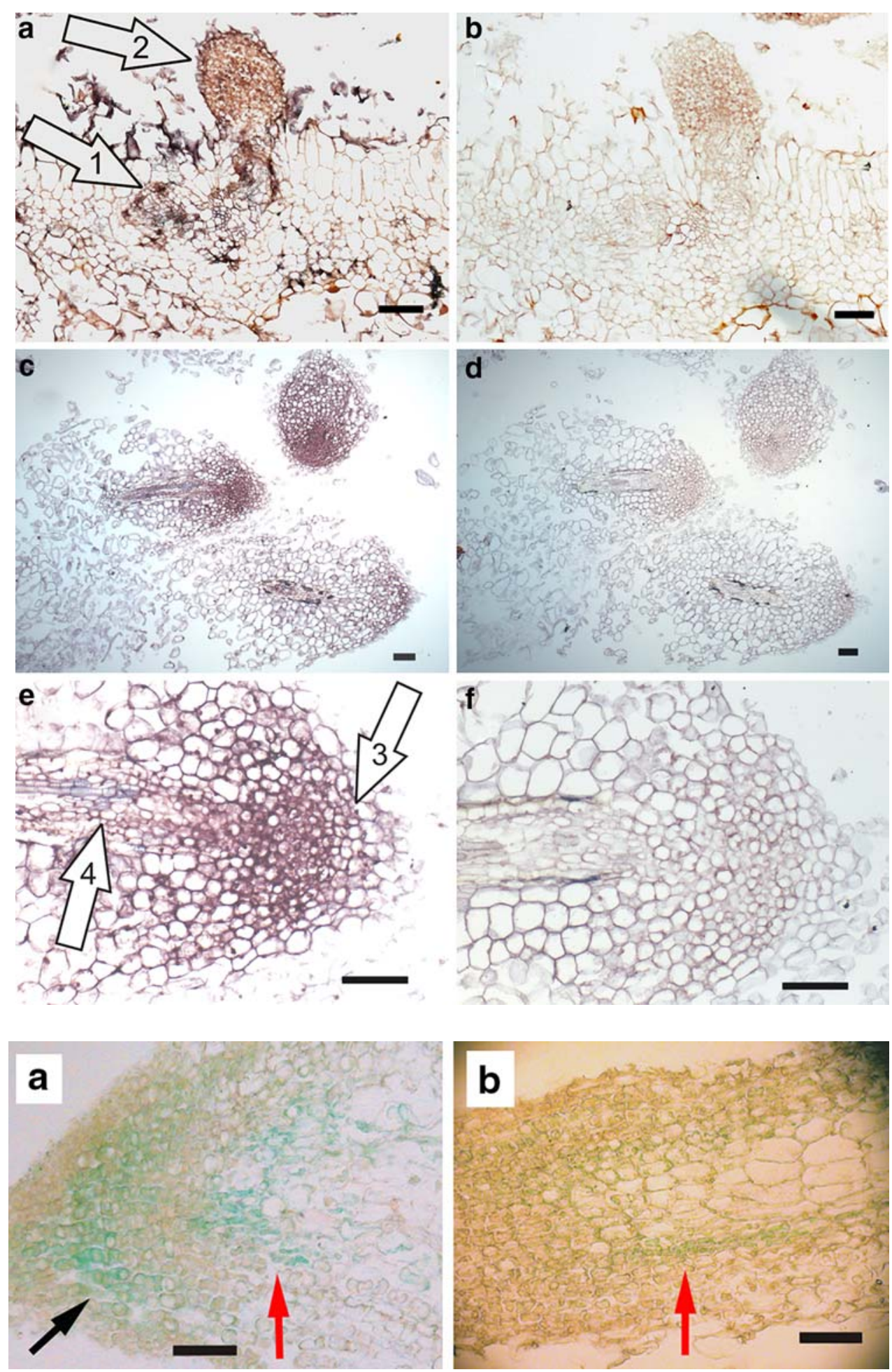

developmental change, thus marking cells as they change into a new developmental pathway (Nolan et al. 2003, 2009). MtSERF 1 expression is evident after about 10 days of culture and is dependent on ethylene as well as auxin and cytokinin. It appears to act as a nexus between the stress of excision and culture (the stress reflected in ethylene synthesis) and the developmental hormones auxin and cytokinin driving cells into SE (Mantiri et al. 2008a, b). Importantly there is some evidence that cytokinin-induced WUS may be necessary for MtSERF1 expression as binding sites for the WUS transcription factor exist in the MtSERF1 promoter region (Mantiri et al. 2008a). It appears that MtSERF1, possibly in conjunction with WUS, is involved in regulating downstream genes required for SE (Mantiri et al. 2008a, b). 
MtSERF1 expression commences earlier than $M t C L V 3$ expression. MtCLV3 likely expresses when stem cells start to be regulated. RNAi studies with MtCLV3 would help resolve this question.

MtWOX5 expression in relation to de novo root formation

MtWOX5, based on the bioinformatics analysis is the putative ortholog of AtWOX5. The expression and in situ data presented here are consistent with this and confirm other recent work on MtWOX5 (Imin et al. 2007).

In-situ hybridisation of the roots induced in culture has suggested that MtWOX5 is expressed in the procambium cells and is associated with the induction of root primordia. It has been shown previously in our laboratory that root primordia were derived from these cells (Rose et al. 2006). In this it appears to have a somewhat similar role to WUS involvement in SE stem cell formation. After the root primordia formed, the meristem showed strong MtWOX5 expression, in what is the quiescent centre/stem cell area that is the source of the root cells and root cap cells. MtWOX 5 expression was clearly auxin-dependent, contrasting with $M t W U S$. The GUS expression studies were not entirely consistent with the hybridisation studies as there are two areas of expression adjacent to the quiescent centre (Fig. 14a) as opposed to the more uniform hybridisation signals in the root tip (Fig. 13c, e). It is also possible that the promoter length, based on the sequence information we had for this study, was insufficient. Also there is the presence of the exogenous auxin associated with an in vitro system. Nevertheless the strong WOX5 expression in the primordium and meristem is clear.

In the intact Medicago plant, Imin et al. (2007) showed that there is low MtWOX5 expression in the root tip compared to root forming calli. This difference is apparent from the in situ studies presented here and suggests a strong auxin response in vitro, and expression outside the quiescent centre associated with the induction and development of the cultured roots. Auxin clearly up-regulates $M t W O X 5$ expression (Fig. 5b) and we have observed in MtWOX5:: GUS expressing meristems that increased auxin concentration increases the area of GUS expression. The Arabidopsis root has a closed meristem (Dolan et al. 1993), while M. truncatula being a legume has an open meristem (Heimsch and Seago 2008). Our study also indicated that there was MtWOX5 expression in the pericycle and procambial tissue of mature roots. It is feasible that this expression was related to a capacity for lateral root formation in planta. Such expression was not reported for Arabidopsis WOX5. The low expression of MtWOX5 in the root tip in planta relative to the in vitro expression was different to BBM (BABY BOOM) and PLT1 (PLETHORA 1) which expressed strongly in $M$. truncatula root-forming calli and root tips in planta (Imin et al. 2007).

MtWOX5 expression is very closely associated with root meristem formation and expresses in the stem cell areas of both the emerging primordium and in the cultured roots (Figs. 13, 14). The expression in the intact root meristem is likely confined to the quiescent centre as in Arabidopsis (Blilou et al. 2005). This may well be due to the close regulation by auxin which is different to the culture system (Gonzali et al. 2005). There is a parallel here with the $M t W U S$ and SE induction as there is initial expression in many cells until the precision of the in planta regulation is set in train. However in vivo and in vitro WOX5 appears to act as a stem cell signal and is intimately associated with stem cell maintenance. RNAi studies would provide more direct evidence. MtWOX5 is also associated with embryo development and as shown recently, there is some overlap with WUS in their developmental roles (Sarkar et al. 2007).

Changes prior to WOX5 expression have not been welldocumented, but ROS production could most likely be an initial event and the regulation of redox is an important consideration in setting up a root meristem (Imin et al. 2007). Other studies in $M$. truncatula also indicate induction of PLETHORA and BABY BOOM (Imin et al. 2007) known to be key players in stem cell maintenance in the Arabidopsis primary meristem (Galinha et al. 2007). However, we do not know the time course of their transcription in relation to $W O X 5$.

Relationship between hormones and gene regulation in SE and root formation

It is apparent that the production of the SEs with their bipolar meristems and the production of the unipolar root meristem have different requirements for the key developmental and stress hormones. Through regulation of specific genes, hormones and morphogens are able to exert regulatory influence. In both cases the culture process has "hijacked" key developmental genes to drive the induction of the in vitro processes. Though these processes are not usual in the M. truncatula life cycle, similar processes do occur in nature. The relationship of ethylene to SE and in vitro root formation is different. In the case of SE ethylene is essential (Mantiri et al. 2008a, b) whereas for in vitro root formation it is inhibitory (Rose et al. 2006), suggesting a priority for reproduction.

MtWUS and MtWOX5 may have a similar function in relation to stem cell induction in vitro

MtWUS and MtWOX5 may have similar functions in stem cell initiation. WUS and WOX5 have been reported to have related roles in maintaining stem cells (Sarkar et al. 2007), 
and also have similar roles in stem cell induction in Medicago. WUS may induce stem cells for somatic embryos with cytokinin being essential, and WOX5, which is partially suppressed by cytokinin, may induce stem cells for root primordium formation with auxin being an essential co-regulator. The requirement for cytokinin and auxin in the regulation of the key genes is of course dependent on the species, genotype and explant type as well as the culture process.

Acknowledgments This work was supported by an Australian Research Council Centre of Excellence grant (CEO348212) to the Australian Research Council Centre of Excellence for Integrative Legume Research.

Open Access This article is distributed under the terms of the Creative Commons Attribution Noncommercial License which permits any noncommercial use, distribution, and reproduction in any medium, provided the original author(s) and source are credited.

\section{References}

Bäurle I, Laux T (2005) Regulation of WUSCHEL transcription in the stem cell niche of the Arabidopsis shoot meristem. Plant Cell 17:2271-2280

Beveridge CB, Mathesius U, Rose RJ, Gresshoff PM (2007) Common regulatory themes in meristem development and whole plant homeostasis. Curr Opin Plant Biol 10:44-51

Blilou I, Xu J, Wildwater M, Willemsen V, Paponov I, Friml J, Heidstra R, Aida M, Palme K, Scheres B (2005) The PIN auxin efflux facilitator network controls growth and patterning in Arabidopsis roots. Nature 433:39-44

Brand U, Fletcher JC, Hobe M, Meyerowitz EM, Simon R (2000) Dependence of stem cell fate in Arabidopsis on a feedback loop regulated by $C L V 3$ activity. Science 289:617-619

Curtis MD, Grossniklaus U (2003) A gateway cloning vector set for high-throughput functional analysis of genes in planta. Plant Physiol 133:462-469

Dolan L, Janmaat K, Willemsen V, Linstead P, Poethig S, Roberts K, Scheres B (1993) Cellular organization of the Arabidopsis thaliana root. Development 119:71-84

Fiers M, Ku KL, Liu C-M (2007) CLE peptide ligands and their role in establishing meristems. Curr Opin Plant Biol 10:39-43

Galinha C, Hofhuis H, Luijten M, Willemsen V, Blilou I, Heidstra R, Scheres B (2007) PLETHORA proteins as dose-dependent master regulators of Arabidopsis root development. Nature 449:10531057

Gallois J-L, Nora FR, Mizukami Y, Sablowski R (2004) WUSCHEL induces shoot stem cell activity and developmental plasticity in the root meristem. Gene Dev 18:375-380

Gonzali S, Novi G, Loreti E, Paolicchi F, Poggi A, Alpi A, Perata P (2005) A turanose-insensitive mutant suggests a role for WOX5 in auxin homeostasis in Arabidopsis thaliana. Plant J 44:633-645

Gordon SP, Heisler MG, Reddy GV, Ohno C, Das P, Meyerowitz EM (2007) Pattern formation during de novo assembly of the Arabidopsis shoot meristem. Development 134:3539-3548

Haecker A, Groß-Hardt R, Geiges B, Sarkar A, Breuninger H, Herrmann M, Laux T (2004) Expression dynamics of WOX genes mark cell fate decisions during early embryonic patterning in Arabidopsis thaliana. Development 131:657-668

Heimsch C, Seago JL (2008) Organization of the root apical meristem in angiosperms. Am J Bot 95:1-21
Huson DH, Richter DC, Rausch C, Dezulian T, Franz M, Rupp R (2007) Dendroscope-an interactive viewer for large phylogenetic trees. BMC Bioinformatics 8:460

Imin N, Nizamidin M, Wu T, Rolfe BG (2007) Factors involved in root formation in Medicago truncatula. J Exp Bot 58:439-451

Leibfried A, To JPC, Busch W, Stehling S, Kehle A, Demar M, Kieber JJ, Lohmann JU (2005) WUSCHEL controls meristem function by direct regulation of cytokinin-inducible response regulators. Nature 438:1172-1175

Mantiri FR, Kurdyukov S, Lohar DP, Sharopova N, Saeed NA, Wang X-D, VandenBosch KA, Rose RJ (2008a) The transcription factor MtSERF1 of the ERF subfamily identified by transcriptional profiling is required for somatic embryogenesis induced by auxin plus cytokinin in Medicago truncatula. Plant Physiol 146:1622-1636

Mantiri FR, Kurdyukov S, Chen S-K, Rose RJ (2008b) The transcription factor MtSERF1 may function as a nexus between stress and development in somatic embryogenesis in Medicago truncatula. Plant Signal Behav 3:498-500

Mayer KFX, Schoof H, Haecker A, Lenhard M, Jürgens G, Laux T (1998) Role of WUSCHEL in regulating stem cell fate in the Arabidopsis shoot meristem. Cell 95:805-815

Mordhorst AP, Voerman KJ, Hartog MV, Meijer EA, van Went J, Koornneef M, de Vries SC (1998) Somatic embryogenesis in Arabidopsis thaliana is facilitated by mutations in genes repressing meristematic cell divisions. Genetics 149:549-563

Müller R, Borghi L, Kwiatkowska D, Laufs P, Simon R (2006) Dynamic and compensatory responses of Arabidopsis shoot and floral meristems to CLV3 signaling. Plant Cell 18:1188-1198

Müller R, Bleckman A, Simon R (2008) The receptor kinase CORYNE of Arabidopsis transmits the stem cell-limiting signal CLAVATA3 independently of CLAVATA1. Plant Cell 20:934-946

Nolan KE, Irwanto RR, Rose RJ (2003) Auxin up-regulates MtSERK1 expression in both Medicago truncatula root-forming and embryogenic cultures. Plant Physiol 133:218-230

Nolan KE, Kurdyukov S, Rose RJ (2009) Expression of the SOMATIC EMBRYOGENESIS RECEPTOR-LIKE KINASE1 (SERK1) gene is associated with developmental change in the life cycle of the model legume Medicago truncatula. J Exp Bot 60:1759-1771

Oelkers K, Goffard N, Weiller GF, Gresshoff PM, Mathesius U, Frickey T (2008) Bioinformatic analysis of the CLE signalling peptide family. BMC Plant Biol 8:1

Ogawa M, Shinohara H, Sakagami Y, Matsubayashi Y (2008) Arabidopsis CLV3 peptide directly binds CLV1 ectodomain. Science 319:294

Page RDM (1996) Tree view: an application to display phylogenetic trees on personal computers. Bioinformatics 12:357-358

Ramakers C, Ruijer JM, Lekanne RH, Deprez L, Moorman AFM (2003) Assumption-free analysis of quantitative real-time polymerase chain reaction (PCR) data. Neurosci Lett 339:62-66

Reddy GV, Meyerowitz EM (2005) Stem-cell homeostasis and growth dynamics can be uncoupled in the Arabidopsis shoot apex. Science 310:663-667

Rose RJ (2008) Medicago truncatula as a model for understanding plant interactions with other organisms, plant development and stress biology: past, present and future. Funct Plant Biol 35:253-264

Rose RJ, Nolan KE (2006) Genetic regulation of somatic embryogenesis with particular reference to Arabidopsis thaliana and Medicago truncatula. In Vitro Cell Dev Biol Plant 42:473-481

Rose RJ, Nolan KE, Bicego L (1999) The development of the highly regenerable seed line Jemalong $2 \mathrm{HA}$ for transformation of Medicago truncatula: implications for regenerability via somatic embryogenesis. J Plant Physiol 155:788-791

Rose RJ, Wang X-D, Nolan KE, Rolfe BG (2006) Root meristems in Medicago truncatula tissue culture arise from vascular-derived procambial-like cells in a process regulated by ethylene. J Exp Bot 57:2227-2235 
Saitou N, Nei M (1987) The neighbour-joining method: a new method for reconstructing phylogenetic trees. Mol Biol Evol 4:406-425

Sarkar AK, Luijten M, Miyashima S, Lenhard M, Hashimoto T, Nakajima K, Scheres B, Heidstra R, Laux T (2007) Conserved factors regulate signalling in Arabidopsis thaliana shoot and root stem cell organizers. Nature 446:811-814

Scheres B (2005) Stem cells: a plant biology perspective. Cell 122:499-504

Shani E, Yanai O, Ori N (2006) The role of hormones in shoot apical meristem function. Curr Opin Plant Biol 9:484-489
Wang JH, Rose RJ, Donaldson BI (1996) Agrobacterium-mediated transformation and expression of foreign genes in Medicago truncatula. Aust J Plant Physiol 23:265-270

Wielopolska A, Townley H, Moore I, Waterhouse P, Helliwell C (2005) A high-throughput inducible RNAi vector for plants. Plant Biotechnol J 3:583-590

Zuo J, Niu Q-W, Frugis G, Chua N-H (2002) The WUSCHEL gene promotes vegetative-to-embryonic transition in Arabidopsis. Plant J 30:349-359 